\title{
FENOLOGIA E PRODUÇÃO DE FRUTOS DE Caryocar brasiliense Cambess. E Enterolobium gummiferum (Mart.) J.F.Macbr. EM DIFERENTES REGIMES DE QUEIMA $^{1}$
}

Renata Françoso², André de Camargo Guaraldo ${ }^{3}$, Manrique Prada ${ }^{4}$, Artur Orelli Paiva5, Estefânia Hofmann Mota $^{5}$ e José Roberto Rodrigues Pinto ${ }^{6}$

\begin{abstract}
RESUMO - O conhecimento dos períodos reprodutivos e vegetativos das plantas é de extrema importância para coletas de sementes e produção de mudas. No Cerrado, as interações da biota com o fogo são de grande interesse, já que esses fatores estão intimamente relacionados e podem ser determinantes na manutenção das populações vegetais. Durante 12 meses, foi estudada a fenologia de duas espécies nativas do Cerrado (Enterolobium gummiferum (Mart.) J.F.Macbr. e Caryocar brasiliense Cambess.) em dois regimes de queima: em sítio mantido sob queima bienal modal no mês de agosto (PBM) e em sítio controle, livre de fogo há 14 anos (PC). Os testes de uniformidade foram realizados para verificar a sazonalidade das fases fenológicas. A seleção de modelos por critério de informação de Akaike foi realizada utilizando as variáveis climáticas da área de estudo para identificar quais delas são melhores preditoras das fases fenológicas. A produção de frutos foi estimada nos dois tratamentos. Praticamente, todas as fenofases apresentaram picos de produtividade, com exceção da brotação foliar de C. brasiliense em PC, queda foliar da mesma espécie nos dois tratamentos e E. gummiferum na PC. Em geral, o comportamento fenológico de ambas as espécies não diferiu de outros estudos. Porém, o fogo retardou a troca de folhas e inibiu as fases reprodutivas de $C$. brasiliense, o que corrobora o comportamento já observado em outras espécies nativas do Cerrado em áreas com incidência de queimadas.
\end{abstract}

Palavras-chave: Cerrado; Orelha-de-macaco; Pequi, sazonalidade.

PHENOLOGY AND FRUIT PRODUCTION OF Caryocar brasiliense Cambess. AND Enterolobium gummiferum (Mart.) J.F.Macbr UNDER DIFFERENT BURN REGIMES

\begin{abstract}
The knowledge of plant reproductive and vegetative periods is extremely important for seed harvesting and seedling production. Interactions between the Cerrado (Brazilian Savanna) biota and fire are of extreme interest because these factors are intimately related and could be determinant in plant populations maintenance. For 12 months, we studied the phenology of two Cerrado native species (Enterolobium gummiferum (Mart.) J.F.Macbr. and Caryocar brasiliense Cambess.) under two fire regimes: the first was burned every two years on August (PBM) in a control site without fire for 14 years (PC). Uniformity tests were made to verify seasonality of each phenological phase. The model selection with Akaike information criterion was made using climatic variables of the study region to identify which variables better describe the phenological phases. Fruit production was estimated for both treatments. Almost every phenophase showed production peaks, except for leaf sprout of $\boldsymbol{C}$. brasiliense in PC and leaf fall of $\boldsymbol{C}$. brasiliense in the two treatments, and E. gummiferum in PC. In general, the phenological behaviour of both species did not differ from other studies. However, fire retarded the leaf renewal and inhibited the reproductive phases of $\boldsymbol{C}$. brasiliense, similar to previous results for other Cerrado native species in regions with burn incidence.
\end{abstract}

Keywords: Cerrado (Brazilian Savanna); Earpod tree; Pequi, seasonality.

\footnotetext{
${ }^{1}$ Recebido em 02.04.2013 aceito para publicação em 27.05.2014.

${ }^{2}$ Programa de Pós-Graduação em Ecologia na Universidade de Brasília, UnB, Brasil. E-mail: <renatafrancoso@yahoo.com.br>. ${ }^{3}$ Departamento de Zoologia, Universidade de Brasília, UNB, Instituto de Ciências Biológicas,Brasil. E-mail: <ac@guaraldo.bio.br>.

${ }^{4}$ Universidade de Brasília, UNB, Brasil. E-mail: <manriqueprada@yahoo.com>.

${ }^{5}$ Mestre em Ciências Florestais, Universidade de Brasília, UNB, Brasil. E-mail: <arturorelli@hotmail.com>e < daliaterra@gmail.com>.

${ }^{6}$ Departamento de Engenharia Florestal, Universidade de Brasília, UNB, Brasil. E-mail: <jrrpinto@unb.br>.
}

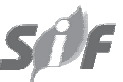

Revista Árvore, Viçosa-MG, v.38, n.4, p.579-590, 2014 


\section{INTRODUÇÃO}

Fenologia é o ramo da Ecologia que estuda eventos biológicos e sua relação com as condições ambientais (BADECK et al., 2004). Este tipo de investigação é importante para subsidiar estudos envolvendo interações de herbivoria, polinização, frugivoria e predação e dispersão de sementes (KLINK et al., 2002). Além disso, são fundamentais no planejamento de coletas de sementes para estudos de fisiologia e germinação, revisões taxonômicas, produção de mudas, plantios, manejo florestal, colheita de frutos e levantamentos florísticos, entre outros (ALENCAR, 1994).

A época em que acontecem os eventos reprodutivos nos vegetais é determinante para o sucesso da população, ao assegurar a sobrevivência e estabelecimento dos indivíduos jovens (FERRAZ et al., 1999). O sincronismo das fenofases se dá por fatores abióticos (ALENCAR, 1994; FERRAZ et al., 1999) e por pressões seletivas de agentes polinizadores e dispersores (ALENCAR, 1994). Nesse sentido, estratégias ecológicas e a filogenia são fatores importantes na determinação das fenofases (FERRAZ et al., 1999; BULHÃO; FIGUEIREDO, 2002; STAGGEMEIER et al., 2010).

Nas formações savânicas do Cerrado, diversas variáveis influenciam a fenologia, como: precipitação, temperatura, insolação, sazonalidade climática e demanda evaporativa (BULHÃO; FIGUEIREDO, 2002; KLINK, 2006; FIGUEIREDO, 2008; VILELA et al., 2008; LENZA; PIRANI et al., 2009; SILVÉRIO; LENZA, 2010; GUILHERME et al., 2011). Adicionalmente, o regime de fogo pode alterar tanto as fenofases quanto a abundância das populações (LOPES et al., 2009) e seu sucesso reprodutivo, tendo efeitos diferenciados sobre cada espécie e cada grupo funcional (SARTORELLI et al., 2007). Assim, em geral, a abundância de espécies lenhosas pode diminuir drasticamente com queimadas frequentes, enquanto o estrato herbáceo é favorecido nessa mesma condição (KLINK et al., 2002).

Incêndios no Cerrado são comuns no período da seca, por ocorrência natural ou de origem antrópica (RODRIGUES, 1996; SATO; MIRANDA, 1996), e são considerados o distúrbio mais comum e antigo nessa vegetação (KLINK et al., 2002). A adaptação de várias espécies ao fogo sugere uma coevolução entre elas, e as respostas morfológicas e fisiológicas variam de acordo com o tipo, a intensidade e a frequência da queima (MIRANDA et al., 2004).
Nesse sentido, estudos fenológicos específicos são necessários para estabelecer o regime de queimadas adequado para a vegetação do Cerrado em ambientes monitorados, de tal forma que seja possível remover o acúmulo de combustível sem alterar significativamente a composição, a estrutura e os processos ecológicos da vegetação (SATO; MIRANDA, 1996).

O objetivo deste estudo foi avaliar a influência dos elementos climáticos e dos regimes de queimadas nos padrões de fenologia vegetativa e reprodutiva das espécies arbóreas Caryocar brasiliense Cambess e Enterolobium gummiferum (Mart.) J.F.Macbr., ambas nativas do Cerrado.

\section{MATERIAL E MÉTODOS}

\subsection{Caracterização da área de estudo}

Este estudo foi realizado na Reserva Ecológica do Roncador (RECOR), localizada em Brasília, DF (1556’ S; 4752’W). A Reserva ocupa uma área de 1.300 ha e está situada a $1.100 \mathrm{~m}$ de altitude (RECOR, 2013). A RECOR é uma das Áreas-Núcleo da Reserva da Biosfera do Cerrado e, juntamente com o Jardim Botânico de Brasília e a Fazenda Água Limpa (FAL/ UnB), forma área de preservação de aproximadamente 10.000 ha, inserida na Área de Proteção Ambiental Gama-Cabeça de Veado (RECOR, 2013).

O período chuvoso na região vai de outubro a abril, com precipitação pluvial média anual de 1.453 mm. As temperaturas na Reserva são elevadas no período chuvoso e amenas na seca, com média anual de $22,0^{\circ} \mathrm{C}$. Os meses mais quentes são setembro e outubro, com temperaturas médias mensais de até $24,0{ }^{\circ} \mathrm{C}$. Junho e julho são os meses mais frios, com temperatura média próxima de $20,0^{\circ} \mathrm{C}$ (RECOR, 2013). Os solos da Reserva são muito diversificados, marcados pela presença de latossolos, cambissolos, plintossolos, gleissolos e organossolos (OLIVEIRA, 2011). As principais fitofisionomias presentes na RECOR são Cerrado sentido restrito, mata de galeria, vereda, campo limpo e campo sujo (PEREIRA; FURTADO, 2011).

\subsection{Amostragem}

O experimento foi realizado nas parcelas bienalmodal (PBM) e controle (PC) do Projeto Fogo (MIRANDA et al., 2011). A PBM ocupa uma área de 
10 ha, com as dimensões de 200 x 500 m, com regime de queimadas em períodos de dois em dois anos no mês de agosto, com a última queima controlada anterior ao experimento, tendo ocorrido em 2004 (MIRANDA et al., 2011). Essa parcela sofreu incêndio acidental em setembro de 2005, abrangendo metade da sua extensão. A PC possui 7,5 ha e até o período do experimento não havia sofrido queima desde 1975. Ambas as parcelas foram estabelecidas em Cerrado típico (PEREIRA; FURTADO, 2011) sobre Latossolo Vermelho (OLIVEIRA, 2011).

\subsection{Fenologia e produção de frutos}

As espécies selecionadas foram pequi (Caryocar brasiliense Cambess., Caryocaraceae) e orelha-demacaco (Enterolobium gummiferum (Mart.) J.F.Macbr., Fabaceae), devido à sua ampla distribuição no Cerrado e por apresentar picos anuais de floração (NEWSTRON et al., 1993; SILVA-JÚNIOR, 2005). Dez indivíduos adultos de cada espécie (com diâmetros a $30 \mathrm{~cm}$ do solo $\geq 5$ $\mathrm{cm}$ ) foram marcados em cada um dos tratamentos (PBM e PC). A coleta de dados seguiu os procedimentos sugeridos por D’eça-Neves e Morellato (2004), tanto o qualitativo (registro da presença ou ausência das fenofases brotação, queda foliar e floração) quanto o quantitativo (número de frutos imaturos e maduros), com observações mensais durante o período de setembro de 2005 a agosto de 2006.

Para verificar a existência de padrão sazonal dos eventos fenológicos, foi realizado o teste de uniformidade de Rayleigh (ZAR, 1999). Também, foram realizados testes de comparação de médias não paramétricos de Qui-quadrado (ZAR, 1999) entre os diferentes tratamentos das mesmas espécies, para verificar a diferença entre os períodos fenológicos. As análises mencionadas foram realizadas no Programa Oriana (KOVACH, 2006). Para comparar a produção de frutos entre os tratamentos, foi realizado o teste não paramétrico de médias de Wilcoxon (ZAR, 1999), considerado o maior número de frutos maduros registrados em cada indivíduo de cada espécie durante o período de estudo.

Foram usados dados climáticos mensais da Estação Meteorológica da RECOR, como: precipitação mensal (mm), temperatura média $\left({ }^{\circ} \mathrm{C}\right)$, luminosidade média (horas e décimos) (RECOR, 2006) e duração das noites (h), disponíveis no sítio do Observatório Naval dos Estados Unidos (http://www.usno.navy.mil). A seleção de modelos por critério de informação de Akaike (AIC) foi realizada no aplicativo SAM (RANGEL et al., 2010), para verificar qual(is) variável(is) climática(s) explica(m) as mudanças nas fases fenológicas das espécies avaliadas.

\section{RESULTADOS}

\subsection{Fases vegetativas}

Foram observados indivíduos em brotação ao longo de todo o período estudado, com concentração maior entre janeiro e fevereiro (Tabela 1; Figura 1), com exceção de C. brasiliense no tratamento PBM, que apresentou reposição da copa de maneira uniforme ao longo do ano. Os modelos selecionados pelo AIC indicaram que a duração das noites explicou, de maneira mais eficiente, a brotação de C. brasiliense nos dois tratamentos (Tabela 2), representando a importância de $61 \%$ na PC, onde a brotação é favorecida quando as noites são mais curtas, mas apenas 35\% na PBM (Tabela 3). Apesar do pico da brotação de E. gummiferum ter sido observado em períodos próximos (janeiro e fevereiro), nos tratamentos PBM e PC houve diferença significativa entre os tratamentos $\left(x^{2}=29,916 \mathrm{p}=\right.$ $0,002)$, em oposição ao observado em $C$. brasiliense $\left(x^{2}=12,32 \mathrm{p}=0,340\right)$.

O pico da ocorrência de folhas maduras, ou seja, a fase entre a brotação e a queda, foi significativo em todos os casos e ocorreu em junho (Tabela 1, Figura 1), precedendo o período de queda foliar. A seleção de modelos pelo AIC indicou a variável duração das noites como mais explicativa para esse evento fenológico nas duas espécies, nos dois tratamentos, incluindo também temperatura média como variável explicativa para essa fenofase de $C$. brasiliense na PBM (Tabela 3). O período de maturidade foliar não diferiu significativamente entre os tratamentos de ambas as espécies (E. gummiferum: $x^{2}=3,299 \mathrm{p}=0,986$; e C. brasiliense: $x^{2}=2,41 \mathrm{p}=$ $0,99)$.

O desfolhamento das copas ocorreu de maneira uniforme ao longo do ano em C. brasiliense, mas foi sazonal em E. gummiferum (PC: agosto; PBM: setembro), embora com baixa concentração (valor de r; Tabela 1), em resposta à duração das noites (Tabela 2, Tabela 3). A data média dessa fenofase não diferiu significativamente entre os tratamentos de ambas as espécies ( $E$. gummiferum: $x^{2}=6,365 \mathrm{p}=0,848$; e $C$. brasiliense: $\left.x^{2}=15,941 \mathrm{p}=0,143\right)$.

Revista Árvore, Viçosa-MG, v.38, n.4, p.579-590, 2014 


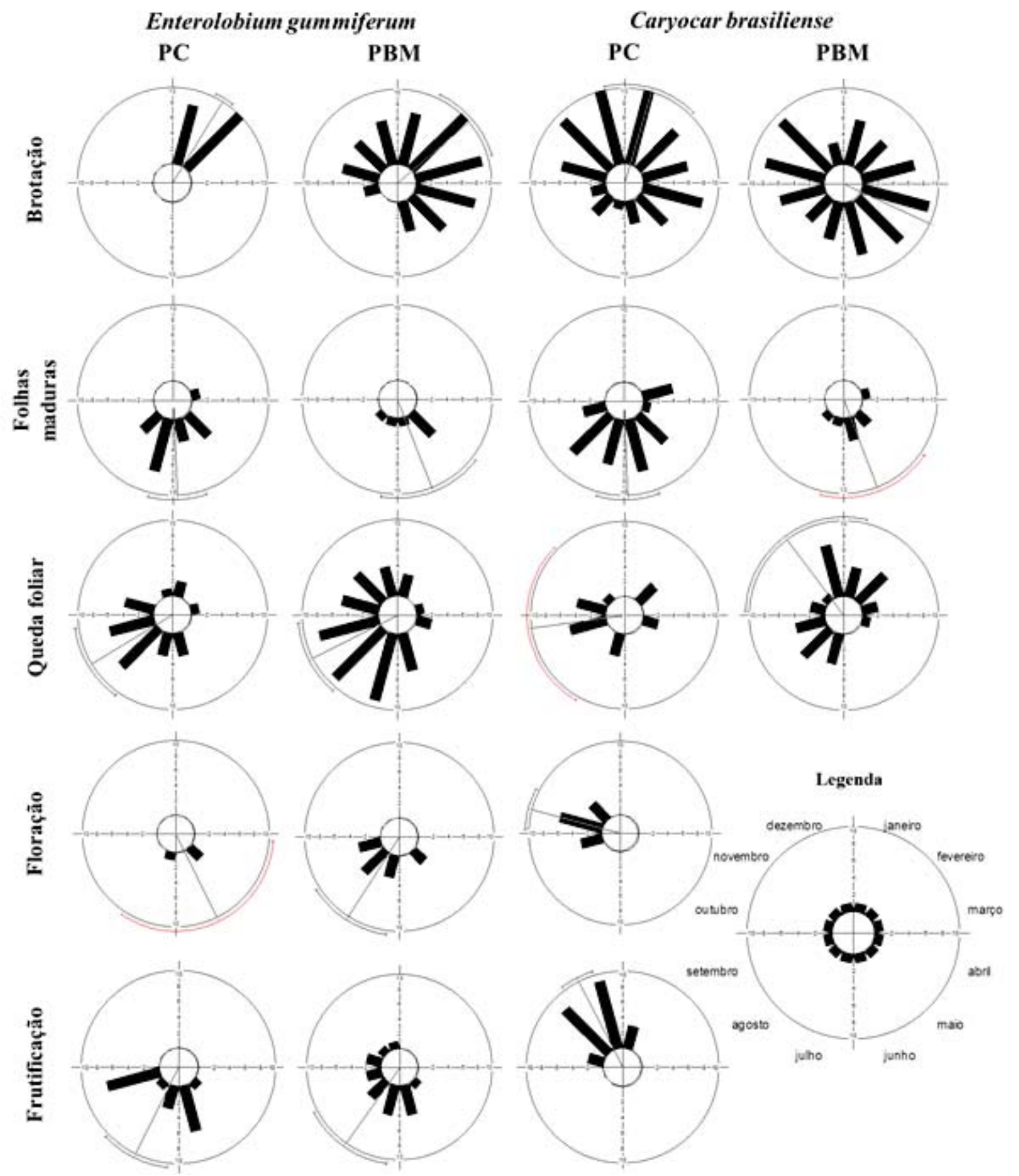

Figura 1 - Distribuição circular das fases fenológicas de Caryocar brasiliense e Enterolobium gummiferum em diferentes regimes de queima na Reserva Ecológica do Roncador, Brasília, DF. Ausência de floração e frutificação de C. brasiliense na PBM. PBM = parcela bienal-modal e PC = parcela controle.

Figure 1 - Circular distribution of phenological phases of Caryocar brasiliense and Enterolobium gummiferum in different burn regimes at Reserva Ecológica do Roncador, Brasília, DF. Absence of flowering and fruiting of C. brasiliense in the PBM. $P B M=$ modal biennial plot, and $P C=$ control plot.

Revista Árvore, Viçosa-MG, v.38, n.4, p.579-590, 2014 
Tabela 1 -Teste de uniformidade da frequência das fenofases de Caryocar brasiliense e Enterolobium gummiferum em diferentes regimes de queima na Reserva Ecológica do Roncado, Brasília - DF. Data media = data media de ocorrência da fenofase; $r$ = comprimento do vetor de concentração; PBM = parcela bienal-modal e PC = parcela controle.

Table 1 - Uniformity test of the frequency of phenophases of Caryocar brasiliense and Enterolobium gummiferum in different burn regimes at the Reserva Ecológica do Roncador. $P B M=$ modal biennial plot; $P C=$ control plot.

\begin{tabular}{|c|c|c|c|c|c|c|c|}
\hline Fenofase & Espécie & Tratamento & $\mathbf{n}$ & Data média & $\mathbf{r}$ & $\mathbf{p}$ & \\
\hline \multirow[t]{4}{*}{ Brotação } & C. brasiliense & PC & 70 & Janeiro & 0.32 & 0.002 & $*$ \\
\hline & & PBM & 77 & Abril & 0.04 & 0.78 & \\
\hline & E. gummiferum & PC & 75 & Janeiro & 0.34 & 0.002 & * \\
\hline & & PBM & 62 & Fevereiro & 0.4 & 0.001 & * \\
\hline \multirow[t]{4}{*}{ Folhas maduras } & C. brasiliense & $\mathrm{PC}$ & 33 & Junho & 0.64 & 0.006 & * \\
\hline & & PBM & 8 & Junho & 0.77 & 0.009 & * \\
\hline & E. gummiferum & PC & 18 & Junho & 0.8 & 0.008 & * \\
\hline & & PBM & 7 & Junho & 0.83 & 0.02 & * \\
\hline \multirow[t]{4}{*}{ Queda foliar } & C. brasiliense & PC & 18 & Setembro & 0.34 & 0.39 & \\
\hline & & PBM & 35 & Novembro & 0.26 & 0.26 & \\
\hline & E. gummiferum & PC & 27 & Agosto & 0.58 & 0.015 & \\
\hline & & PBM & 51 & Setembro & 0.46 & 0.004 & * \\
\hline \multirow[t]{4}{*}{ Floração } & C. brasiliense & PC & 12 & Outubro & 0.93 & 0.01 & * \\
\hline & & PBM & 1 & Dezembro & - & - & \\
\hline & E. gummiferum & PC & 3 & Junho & 0.88 & 0.37 & \\
\hline & & PBM & 12 & Agosto & 0.78 & 0.02 & * \\
\hline \multirow[t]{4}{*}{ Frutificação } & C. brasiliense & $\mathrm{PC}$ & 22 & Dezembro & 0.91 & 0.001 & $*$ \\
\hline & & PBM & 0 & - & - & - & \\
\hline & E. gummiferum & PC & 18 & Julho & 0.74 & 0.07 & * \\
\hline & & PBM & 18 & Agosto & 0.6 & 0.001 & * \\
\hline
\end{tabular}

\subsection{Fases reprodutivas}

Os baixos registros de floração de $C$. brasiliense na PBM $(\mathrm{N}=1)$ e de E. gummiferum na PC $(\mathrm{N}=3)$ não permitiram registrar o período predominante dessa fenofase nas duas situações. Caryocar brasiliense na PC apresentou floração entre setembro e novembro, com maior ocorrência em outubro (Tabela 1 e Figura 1). Pelo AIC, o aumento da temperatura média foi a melhor preditora da floração de C. brasiliense na PC (Tabela 2 e Tabela 3).

Na PBM, E. gummiferum floresceu entre maio e setembro, com pico em agosto (Tabela 1 e Figura 1). A melhor variável ambiental preditora da floração de E. gummiferum na PBM foi a precipitação total (Tabela 2 e Tabela 3), indicando que a baixa pluviosidade estimula a floração da espécie. Entretanto, não houve diferença significativa no período de floração de E. gummiferum entre os tratamentos (E. gummiferum: $x^{2}=4,062$ $\mathrm{p}=0,968)$.
Não foram registrados indivíduos de $C$. brasiliense frutificando na PBM. No tratamento PC, esse evento ocorreu entre outubro e janeiro, com pico de ocorrência em dezembro (Tabela 1 e Figura 1). Dois modelos explicaram, de maneira similar, a floração de $C$. brasiliense na PC, ambos com apenas uma variável (Tabela 2 e Tabela 3). O primeiro indicou a precipitação total e o segundo, a luminosidade média como principais preditoras da frutificação da espécie.

Enterolobium gummiferum apresentou frutificação entre maio e setembro (maior concentração em julho) na PC e entre maio e dezembro (maior concentração em agosto) na PBM, tendo sido registradas nas mesmas frequências de indivíduos frutificando em ambos os tratamentos (Tabela 1). Tanto na PC quanto na PBM, a melhor variável preditora foi a precipitação total (Tabela 2 e Tabela 3), indicando maior frequência de indivíduos frutificando no período de menor precipitação. Não houve diferença no período de frutificação entre os tratamentos (E. gummiferum: $x^{2}=8,321 \mathrm{p}=0,684$ ). 
Tabela 2 - Modelos selecionados pelo Critério de Informação Akaike para cada fenofase de Caryocar brasiliense e Enterolobium gummiferum em diferentes regimes de queima na Reserva Ecológica do Roncador, Brasília, DF. PBM = parcela bienal-modal; PC = parcela controle; FF = Fenofase; e Variáveis: 1 = Precipitação mensal (mm), 2 = Temperatura média $\left({ }^{\circ} \mathrm{C}\right), 3$ = Luminosidade média e 4 = Duração das noites $(\mathrm{h})$.

Table 2 - Models selected by Akaike information criterion for each phenophase of Caryocar brasiliense and Enterolobium gummiferum under different burn regimes at Reserva Ecológica do Roncador, Brasília, DF. PBM = modal biennial plot; $P C=$ control plot $; F F=$ Phenophase; Variables: $1=$ Monthly precipitation $(\mathrm{mm}), 2=$ Mean temperature $\left({ }^{\circ} \mathrm{C}\right), 3=$ Average brightness and $4=$ Duration of nights $(h)$.

\begin{tabular}{|c|c|c|c|c|c|c|c|c|c|c|}
\hline FF & C. brasiliense & Variáveis & $\mathrm{r}^{2}$ & AICc & $\begin{array}{l}\text { Delta } \\
\text { AICc }\end{array}$ & E. gummiferum & Var & $\mathrm{r}^{2}$ & AICc & $\begin{array}{l}\text { Delta } \\
\text { AICc }\end{array}$ \\
\hline \multirow[t]{8}{*}{ Brotação } & \multirow[t]{6}{*}{$\mathrm{PC}$} & 4 & 0,613 & 57,767 & 0,000 & \multirow[t]{2}{*}{ PC } & 3 & 0,634 & 60,832 & 0,000 \\
\hline & & 1 & 0,585 & 58,619 & 0,853 & & 1 & 0,560 & 63,044 & 2,212 \\
\hline & & 1,4 & 0,698 & 59,490 & 1,724 & \multirow[t]{6}{*}{ PBM } & 1 & 0,379 & 64,527 & 0,000 \\
\hline & & 3 & 0,531 & 60,083 & 2,316 & & 3 & 0,337 & 65,313 & 0,786 \\
\hline & & 3,4 & 0,647 & 61,374 & 3,607 & & 4 & 0,206 & 67,478 & 2,951 \\
\hline & & 2,4 & 0,643 & 61,526 & 3,759 & & & & & \\
\hline & \multirow[t]{2}{*}{ PBM } & 4 & 0,041 & 57,269 & 0,000 & & & & & \\
\hline & & 2 & 0,013 & 57,613 & 0,345 & & & & & \\
\hline \multirow[t]{7}{*}{ Folhas maduras } & \multirow[t]{3}{*}{$\mathrm{PC}$} & 4 & 0,660 & 54,824 & 0,000 & \multirow[t]{4}{*}{ PC } & 4 & 0,609 & 50,472 & 0,000 \\
\hline & & 2,3 & 0,744 & 56,157 & 1,332 & & 2,4 & 0,724 & 51,036 & 0,564 \\
\hline & & 2,4 & 0,708 & 57,746 & 2,922 & & 2,3 & 0,716 & 51,353 & 0,881 \\
\hline & \multirow[t]{4}{*}{ PBM } & 2,4 & 0,745 & 29,934 & 0,000 & & 1,2 & 0,664 & 53,370 & 2,898 \\
\hline & & 4 & 0,602 & 30,573 & 0,639 & \multirow[t]{3}{*}{ PBM } & 4 & 0,420 & 39,127 & 0,000 \\
\hline & & 2,3 & 0,712 & 31,425 & 1,490 & & 3 & 0,245 & 42,295 & 3,167 \\
\hline & & & & & & & 2 & 0,192 & 43,103 & 3,976 \\
\hline \multirow[t]{10}{*}{ Queda foliar } & \multirow[t]{3}{*}{$\mathrm{PC}$} & $1,2,3$ & 0,780 & 49,381 & 0,000 & \multirow[t]{10}{*}{ PC } & 3 & 0,379 & 57,474 & 0,000 \\
\hline & & $1,2,3,4$ & 0,894 & 49,435 & 0,054 & & 1 & 0,361 & 57,807 & 0,332 \\
\hline & & 2 & 0,313 & 52,058 & 2,677 & & 3,4 & 0,520 & 59,088 & 1,613 \\
\hline & \multirow[t]{7}{*}{ PBM } & 4 & 0,268 & 57,567 & 0,000 & & 2,3 & 0,512 & 59,295 & 1,820 \\
\hline & & 3 & 0,067 & 60,473 & 2,906 & & 1,2 & 0,485 & 59,951 & 2,476 \\
\hline & & 2 & 0,040 & 60,814 & 3,247 & & 1,4 & 0,429 & 61,180 & 3,706 \\
\hline & & 1 & 0,037 & 60,847 & 3,280 & & 1 & 0,229 & 66,982 & 0,000 \\
\hline & & 1,4 & 0,332 & 61,182 & 3,615 & & 3 & 0,164 & 67,943 & 0,961 \\
\hline & & 3,4 & 0,324 & 61,329 & 3,762 & & 4 & 0,049 & 69,488 & 2,506 \\
\hline & & & & & & & 2 & $<.001$ & 70,096 & 3,114 \\
\hline \multirow[t]{7}{*}{ Floração } & \multirow[t]{7}{*}{$\mathrm{PC}$} & 2 & 0,535 & 48,891 & 0,000 & \multirow[t]{4}{*}{ PC } & 4 & 0,359 & 25,256 & 0,000 \\
\hline & & 2,4 & 0,587 & 52,195 & 3,303 & & 2 & 0,160 & 28,504 & 3,248 \\
\hline & & & & & & & 3 & 0,122 & 29,031 & 3,775 \\
\hline & & & & & & & 3,4 & 0,397 & 29,250 & 3,994 \\
\hline & & & & & & \multirow[t]{3}{*}{ PBM } & 1 & 0,386 & 46,476 & 0,000 \\
\hline & & & & & & & 3 & 0,336 & 47,425 & 0,949 \\
\hline & & & & & & & 4 & 0,200 & 49,652 & 3,176 \\
\hline \multirow[t]{8}{*}{ Frutificação } & \multirow[t]{8}{*}{ PC } & 1 & 0,494 & 62,279 & 0,000 & \multirow[t]{4}{*}{ PC } & 1 & 0,346 & 58,949 & 0,000 \\
\hline & & 3 & 0,491 & 62,357 & 0,078 & & 4 & 0,277 & 60,151 & 1,202 \\
\hline & & 4 & 0,406 & 64,209 & 1,931 & & 3 & 0,188 & 61,543 & 2,594 \\
\hline & & 1,4 & 0,531 & 66,091 & 3,812 & & 1,3 & 0,421 & 62,199 & 3,250 \\
\hline & & & & & & \multirow[t]{4}{*}{ PBM } & 1 & 0,440 & 44,914 & 0,000 \\
\hline & & & & & & & 3 & 0,436 & 44,982 & 0,068 \\
\hline & & & & & & & 4 & 0,418 & 45,370 & 0,456 \\
\hline & & & & & & & 1,2 & 0,527 & 47,584 & 2,670 \\
\hline
\end{tabular}

\subsection{Produção de frutos}

A produção de frutos de $C$. brasiliense foi significativamente maior na PC do que na PBM (W
$=5 ; \mathrm{p}<0,001 . \mathrm{PC}=24 \pm 15 ; \mathrm{PBM}=0 \pm 0$ ). A produção de E. gummiferum não diferiu significativamente entre os tratamentos $(\mathrm{W}=67 ; \mathrm{p}=0,21$. $\mathrm{PC}=12 \pm 9$; $\mathrm{PBM}$ $=34 \pm 46)$.

Revista Árvore, Viçosa-MG, v.38, n.4, p.579-590, 2014 
Fenologia e produção de frutos de...

Tabela 3 - Importância das variáveis climáticas dos modelos selecionados pelo Critério de Informação de Akaike para diferentes fenofases (FF), espécies (spp.) e regimes de queima (RQ); PBM = parcela bienal-modal; e PC = parcela controle.

Table 3 - Importance of climatic variables of models selected by Akaike Information Criterion for different phenophases $(F F)$, species (spp.) and burn regimes (RQ); PBM = modal biannual plot; and PC = control plot.

\begin{tabular}{|c|c|c|c|c|c|c|c|c|c|c|}
\hline$\overline{\mathrm{FF}}$ & Spp. & RQ & Variável & Import. & Coef. & $\begin{array}{l}\text { Coef. } \\
\text { padrão }\end{array}$ & $\begin{array}{c}\text { Erro } \\
\text { padrão }\end{array}$ & $\mathrm{t}$ & $\begin{array}{c}95 \% \\
\text { Inferior }\end{array}$ & $\begin{array}{c}95 \% \\
\text { Superior }\end{array}$ \\
\hline \multirow[t]{20}{*}{ Brotação } & \multirow[t]{10}{*}{ C. brasiliense } & \multirow[t]{5}{*}{$\mathrm{PC}$} & Constante & & 25,731 & 0,000 & 19,147 & 1,344 & $-11,796$ & 63,259 \\
\hline & & & Precipitação Total & 0,430 & 0,015 & 0,632 & 0,003 & 4,928 & 0,009 & 0,021 \\
\hline & & & Temperatura média & 0,102 & $-0,105$ & $-0,059$ & 0,049 & $-2,148$ & $-0,200$ & $-0,009$ \\
\hline & & & Luminosidade média & 0,209 & $-0,708$ & $-0,503$ & 0,116 & $-6,119$ & $-0,934$ & $-0,481$ \\
\hline & & & Duração das noites & 0,609 & $-2,705$ & $-0,692$ & 0,634 & $-4,267$ & $-3,947$ & $-1,462$ \\
\hline & & \multirow[t]{5}{*}{ PBM } & Constante & & 2,743 & 0,000 & 7,817 & 0,351 & $-12,578$ & 18,065 \\
\hline & & & Precipitação Total & 0,264 & $<.001$ & 0,021 & 0,002 & 0,204 & $-0,003$ & 0,003 \\
\hline & & & Temperatura média & 0,287 & 0,154 & 0,138 & 0,104 & 1,470 & $-0,051$ & 0,358 \\
\hline & & & Luminosidade média & 0,270 & $-0,047$ & $-0,054$ & 0,096 & $-0,492$ & $-0,237$ & 0,142 \\
\hline & & & Duração das noites & 0,351 & 0,662 & 0,272 & 0,314 & 2,110 & 0,047 & 1,277 \\
\hline & \multirow[t]{10}{*}{ E. gummiferum } & \multirow[t]{5}{*}{$\mathrm{PC}$} & Constante & & 13,191 & 0,000 & 7,732 & 1,706 & $-1,963$ & 28,345 \\
\hline & & & Precipitação Total & 0,288 & 0,017 & 0,600 & 0,003 & 5,870 & 0,011 & 0,023 \\
\hline & & & Temperatura média & 0,102 & 0,252 & 0,121 & 0,045 & 5,542 & 0,163 & 0,341 \\
\hline & & & Luminosidade média & 0,726 & $-1,285$ & $-0,782$ & 0,274 & $-4,686$ & $-1,822$ & $-0,748$ \\
\hline & & & Duração das noites & 0,129 & $-1,400$ & $-0,307$ & 0,231 & $-6,053$ & $-1,853$ & $-0,947$ \\
\hline & & \multirow[t]{5}{*}{ PBM } & Constante & & 7,752 & 0,000 & 8,810 & 0,880 & $-9,515$ & 25,019 \\
\hline & & & Precipitação Total & 0,537 & 0,015 & 0,607 & 0,004 & 3,840 & 0,008 & 0,023 \\
\hline & & & Temperatura média & 0,112 & 0,184 & 0,099 & 0,060 & 3,046 & 0,066 & 0,303 \\
\hline & & & Luminosidade média & 0,376 & $-0,771$ & $-0,524$ & 0,188 & $-4,110$ & $-1,139$ & $-0,403$ \\
\hline & & & Duração das noites & 0,173 & $-1,124$ & $-0,275$ & 0,282 & $-3,990$ & $-1,676$ & $-0,572$ \\
\hline \multirow{20}{*}{$\begin{array}{l}\text { Queda } \\
\text { foliar }\end{array}$} & \multirow[t]{10}{*}{ C. brasiliense } & \multirow[t]{5}{*}{$\mathrm{PC}$} & Constante & & $-9,629$ & 0,000 & 12,405 & $-0,776$ & $-33,943$ & 14,685 \\
\hline & & & Precipitação Total & 0,847 & $-0,023$ & $-1,600$ & 0,006 & $-4,011$ & $-0,034$ & $-0,012$ \\
\hline & & & Temperatura média & 0,905 & 0,695 & 0,658 & 0,205 & 3,394 & 0,294 & 1,097 \\
\hline & & & Luminosidade média & 0,799 & $-1,338$ & $-1,608$ & 0,345 & $-3,876$ & $-2,014$ & $-0,661$ \\
\hline & & & Duração das noites & 0,402 & 1,409 & 0,609 & 0,263 & 5,359 & 0,894 & 1,925 \\
\hline & & \multirow[t]{5}{*}{ PBM } & Constante & & 15,656 & 0,000 & 13,601 & 1,151 & $-11,001$ & 42,313 \\
\hline & & & Precipitação Total & 0,197 & $-0,001$ & $-0,073$ & 0,002 & $-0,778$ & $-0,004$ & 0,002 \\
\hline & & & Temperatura média & 0,169 & 0,153 & 0,119 & 0,073 & 2,104 & 0,010 & 0,296 \\
\hline & & & Luminosidade média & 0,213 & $-0,039$ & $-0,038$ & 0,108 & $-0,359$ & $-0,250$ & 0,173 \\
\hline & & & Duração das noites & 0,672 & $-1,651$ & $-0,586$ & 0,622 & $-2,656$ & $-2,869$ & $-0,433$ \\
\hline & \multirow[t]{10}{*}{ E. gummiferum } & \multirow[t]{5}{*}{ PC } & Constante & & 0,478 & 0,000 & 9,570 & 0,050 & $-18,279$ & 19,234 \\
\hline & & & Precipitação Total & 0,409 & $-0,011$ & $-0,600$ & 0,002 & $-4,548$ & $-0,016$ & $-0,006$ \\
\hline & & & Temperatura média & 0,251 & 0,492 & 0,354 & 0,088 & 5,569 & 0,319 & 0,665 \\
\hline & & & Luminosidade média & 0,570 & 0,794 & 0,724 & 0,223 & 3,564 & 0,357 & 1,231 \\
\hline & & & Duração das noites & 0,222 & $-1,232$ & $-0,404$ & 0,317 & $-3,891$ & $-1,853$ & $-0,612$ \\
\hline & & \multirow[t]{5}{*}{ PBM } & Constante & & 3,517 & 0,000 & 8,612 & 0,408 & $-13,362$ & 20,396 \\
\hline & & & Precipitação Total & 0,511 & $-0,013$ & $-0,512$ & 0,004 & $-2,985$ & $-0,021$ & $-0,004$ \\
\hline & & & Temperatura média & 0,157 & 0,053 & 0,028 & 0,092 & 0,576 & $-0,127$ & 0,233 \\
\hline & & & Luminosidade média & 0,330 & 0,501 & 0,343 & 0,193 & 2,589 & 0,122 & 0,880 \\
\hline & & & Duração das noites & 0,199 & 0,167 & 0,041 & 0,347 & 0,481 & $-0,513$ & 0,846 \\
\hline \multirow{4}{*}{$\begin{array}{l}\text { Folhas } \\
\text { maduras }\end{array}$} & \multirow[t]{4}{*}{ C. brasiliense } & \multirow[t]{4}{*}{$\mathrm{PC}$} & Constante & & $-15,502$ & 0,000 & 21,702 & $-0,714$ & $-58,038$ & 27,033 \\
\hline & & & Precipitação Total & 0,103 & $-0,003$ & $-0,115$ & 0,001 & $-2,512$ & $-0,005$ & $<.001$ \\
\hline & & & Temperatura média & 0,391 & $-0,694$ & $-0,412$ & 0,138 & $-5,017$ & $-0,966$ & $-0,423$ \\
\hline & & & Luminosidade média & 0,377 & 0,813 & 0,612 & 0,137 & 5,952 & 0,545 & 1,081 \\
\hline
\end{tabular}


Tabela 3 - Cont.

Table 3 - Cont.

\begin{tabular}{|c|c|c|c|c|c|c|c|c|c|c|}
\hline & & & Duração das noites & 0,665 & 2,818 & 0,763 & 0,548 & 5,139 & 1,743 & 3,892 \\
\hline & & PBM & Constante & & $-2,907$ & 0,000 & 7,620 & $-0,381$ & $-17,841$ & 12,027 \\
\hline & & & Precipitação Total & 0,132 & 0,002 & 0,288 & $<.001$ & 4,698 & 0,001 & 0,003 \\
\hline & & & Temperatura média & 0,654 & $-0,289$ & $-0,509$ & 0,080 & $-3,597$ & $-0,446$ & $-0,131$ \\
\hline & & & Luminosidade média & 0,268 & 0,232 & 0,520 & 0,042 & 5,591 & 0,151 & 0,314 \\
\hline & & & Duração das noites & 0,719 & 0,857 & 0,690 & 0,216 & 3,968 & 0,434 & 1,280 \\
\hline & E. gummiferum & PC & Constante & & $-6,367$ & 0,000 & 18,525 & $-0,344$ & $-42,676$ & 29,941 \\
\hline & & & Precipitação Total & 0,141 & $-0,006$ & $-0,314$ & $<.001$ & $-6,413$ & $-0,007$ & $-0,004$ \\
\hline & & & Temperatura média & 0,590 & $-0,653$ & $-0,498$ & 0,170 & $-3,838$ & $-0,987$ & $-0,320$ \\
\hline & & & Luminosidade média & 0,284 & 0,494 & 0,478 & 0,078 & 6,299 & 0,340 & 0,647 \\
\hline & & & Duração das noites & 0,652 & 2,013 & 0,701 & 0,441 & 4,561 & 1,148 & 2,878 \\
\hline & & $\overline{\mathrm{PBM}}$ & Constante & & $-6,605$ & 0,000 & 7,383 & $-0,895$ & $-21,076$ & 7,866 \\
\hline & & & Precipitação Total & 0,147 & $<.001$ & $-0,080$ & $<.001$ & $-1,154$ & $-0,002$ & $<.001$ \\
\hline & & & Temperatura média & 0,202 & $-0,226$ & $-0,337$ & 0,040 & $-5,580$ & $-0,305$ & $-0,146$ \\
\hline & & & Luminosidade média & 0,214 & 0,203 & 0,383 & 0,046 & 4,405 & 0,112 & 0,293 \\
\hline & & & Duração das noites & 0,698 & 0,954 & 0,650 & 0,278 & 3,436 & 0,410 & 1,498 \\
\hline loração & C. brasiliense & PC & Constante & & $-18,548$ & 0,000 & 7,448 & $-2,490$ & $-33,146$ & $-3,950$ \\
\hline & & & Precipitação Total & 0,092 & $-0,001$ & $-0,080$ & $<0,001$ & $-3,225$ & $-0,002$ & $<0,001$ \\
\hline & & & Temperatura média & 0,976 & 0,846 & 0,751 & 0,247 & 3,418 & 0,361 & 1,330 \\
\hline & & & Luminosidade média & 0,092 & 0,009 & 0,010 & 0,024 & 0,378 & $-0,038$ & 0,057 \\
\hline & & & Duração das noites & 0,157 & 0,634 & 0,257 & 0,110 & 5,789 & 0,420 & 0,849 \\
\hline & E. gummiferum & PC & Constante & & $-3,495$ & 0,000 & 4,042 & $-0,865$ & $-11,417$ & 4,427 \\
\hline & & & Precipitação Total & 0,160 & $<0,001$ & $-0,036$ & $<0,001$ & $-0,487$ & $<0,001$ & $<0,001$ \\
\hline & & & Temperatura média & 0,204 & $-0,114$ & $-0,318$ & 0,023 & $-5,007$ & $-0,158$ & $-0,069$ \\
\hline & & & Luminosidade média & 0,181 & 0,021 & 0,076 & 0,025 & 0,844 & $-0,028$ & 0,071 \\
\hline & & & Duração das noites & 0,714 & 0,498 & 0,636 & 0,166 & 2,993 & 0,172 & 0,824 \\
\hline & & $\overline{\mathrm{PBM}}$ & Constante & & $-0,275$ & 0,000 & 4,083 & $-0,067$ & $-8,278$ & 7,727 \\
\hline & & & Precipitação Total & 0,559 & $-0,007$ & $-0,617$ & 0,002 & $-3,781$ & $-0,011$ & $-0,004$ \\
\hline & & & Temperatura média & 0,104 & $-0,007$ & $-0,008$ & 0,027 & $-0,248$ & $-0,059$ & 0,046 \\
\hline & & & Luminosidade média & 0,364 & 0,360 & 0,516 & 0,088 & 4,074 & 0,187 & 0,533 \\
\hline & & & Duração das noites & 0,169 & 0,507 & 0,262 & 0,132 & 3,841 & 0,248 & 0,766 \\
\hline Frutificação & C. brasiliense & PC & Constante & & 10,262 & 0,000 & 12,123 & 0,847 & $-13,497$ & 34,022 \\
\hline & & & Precipitação Total & 0,454 & 0,017 & 0,649 & 0,003 & 4,982 & 0,010 & 0,023 \\
\hline & & & Temperatura média & 0,105 & $-0,244$ & $-0,129$ & 0,055 & $-4,416$ & $-0,352$ & $-0,136$ \\
\hline & & & Luminosidade média & 0,429 & $-0,961$ & $-0,647$ & 0,187 & $-5,146$ & $-1,326$ & $-0,595$ \\
\hline & & & Duração das noites & 0,248 & $-2,110$ & $-0,511$ & 0,355 & $-5,935$ & $-2,806$ & $-1,413$ \\
\hline & E. gummiferum & PC & Constante & & $-2,187$ & 0,000 & 10,957 & $-0,200$ & $-23,662$ & 19,288 \\
\hline & & & Precipitação Total & 0,581 & $-0,013$ & $-0,677$ & 0,004 & $-3,071$ & $-0,022$ & $-0,005$ \\
\hline & & & Temperatura média & 0,135 & $-0,245$ & $-0,170$ & 0,058 & $-4,208$ & $-0,359$ & $-0,131$ \\
\hline & & & Luminosidade média & 0,234 & $-0,077$ & $-0,067$ & 0,189 & $-0,405$ & $-0,447$ & 0,294 \\
\hline & & & Duração das noites & 0,314 & 1,498 & 0,474 & 0,320 & 4,677 & 0,870 & 2,126 \\
\hline & & PBM & Constante & & $-2,165$ & 0,000 & 6,840 & $-0,316$ & $-15,572$ & 11,242 \\
\hline & & & Precipitação Total & 0,410 & $-0,007$ & $-0,602$ & 0,001 & $-4,956$ & $-0,010$ & $-0,004$ \\
\hline & & & Temperatura média & 0,175 & $-0,237$ & $-0,272$ & 0,039 & $-6,125$ & $-0,312$ & $-0,161$ \\
\hline & & & Luminosidade média & 0,382 & 0,408 & 0,597 & 0,080 & 5,124 & 0,252 & 0,565 \\
\hline & & & Duração das noites & 0,319 & 1,047 & 0,551 & 0,188 & 5,560 & 0,678 & 1,417 \\
\hline
\end{tabular}

\section{DISCUSSÃO}

\subsection{Fenofases vegetativas}

A brotação foliar pôde ser observada em ambas as espécies durante todo o período estudado, com maior frequência entre janeiro e fevereiro. Em estudo fenológico de 19 espécies arbóreas de Cerrado sentido restrito na RECOR, foi observado predomínio de indivíduos em fase de brotação foliar na transição entre os períodos de seca e chuva, em setembro e outubro

Revista Árvore, Viçosa-MG, v.38, n.4, p.579-590, 2014 
(LENZA; KLINK, 2006), período também registrado em outros estudos no Cerrado (PIRANI, 2009; SILVÉRIO; LENZA, 2010). Em estudo específico de C. brasiliense, Vilela et al. (2008) registraram brotação ao longo de quase todo o ano, com exceção dos meses de junho e julho, mas com maior frequência também na transição entre os períodos de seca e chuva, assim como observado nessa espécie na PBM. Aparentemente, a brotação nas duas espécies estudadas não apresentou picos acentuados característicos.

As folhas atingem a maturidade em junho, respondendo, principalmente, ao aumento da duração das noites. No Cerrado, a dormência das plantas coincide com os dias mais curtos e não com a restrição hídrica, já que a falta de água não é considerada fator limitante para as espécies adaptadas à sazonalidade climática (LENZA; KLINK, 2006; FIGUEIREDO, 2008; SILVÉRIO; LENZA, 2010). Essa fase precede à queda foliar de E. gummiferum nos meses seguintes na transição entre a seca e a chuva. Esse padrão coincide com o observado em diversas espécies decíduas ou breve-decíduas do Cerrado (LENZA; KLINK, 2006; VILELA et al., 2008). A caducifolia apresentou comportamento complexo diante da variação climática, sendo as variáveis mais importantes a luminosidade média e a precipitação média. Não foi observada perda total das folhas em C. brasiliense, porém essa espécie é considerada caducifólia por outros autores (VILELA et al., 2008). Como para o Cerrado sentido restrito são consideradas espécies breve-decíduas - aquelas que apresentam caducifolia durante não mais que duas semanas (LENZA; KLINK, 2006) -, é possível que os intervalos entre as observações fenológicas de um mês, como adotado neste estudo, podem não ter captado essa fenofase para o pequi.

\subsection{Fenofases reprodutivas}

O incêndio acidental ocorrido na PBM em setembro de 2005 pode ter causado a ausência de floração de C. brasiliense, já que a espécie floresceu a partir desse mês na PC. O período de floração registrado em $C$. brasiliense coincide com o relatado por Silva-Júnior (2005), que citou a floração de pequi em áreas de Cerrado sentido restrito entre os meses de junho a janeiro. Vilela et al. (2008) também registraram o período reprodutivo durante o período chuvoso, que coincide com o período de maior temperatura média. De fato, a temperatura média foi selecionada como melhor modelo preditor para floração de C. brasiliense. A frutificação de C. brasiliense durante o período chuvoso (com pico em dezembro) está de acordo com o registrado por outros autores (SILVA-JÚNIOR, 2005; PIRANI et al., 2009). Quanto à produção de frutos, tanto a precipitação total quanto a luminosidade média influenciaram o período de frutificação de C. brasiliense. O padrão reprodutivo de $C$. brasiliense coincide com o observado em espécies sempre-verdes, as quais florescem na seca e produzem pequenas quantidades de sementes grandes em frutos carnosos (FIGUEIREDO, 2008), que são mais abundantes no período chuvoso (BATALHA; MANTOVANI, 2000).

O período de floração observado em E. gummiferum em agosto e setembro também o foi por Silva-Júnior (2005) e pode ser caracterizado como anual precoce, ou seja, ocorre anualmente no inverno (FIGUEIREDO, 2008). O padrão anual foi também verificado por Lenza e Klink (2006) em 17 das 19 espécies estudadas em Cerrado sentido restrito, predominantemente no período seco (anual precoce) ou na transição entre o período da seca e o das águas (anual intermediária).

Enterolobium gummiferum também apresenta síndrome de dispersão zoocórica. Sua frutificação durante a seca é segura pelo fato de o seu fruto ser seco, mantendo as sementes viáveis por mais tempo (ROCHA; SILVA, 2002) até a chegada das chuvas, quando o estabelecimento das plântulas é mais provável.

\subsection{Regime de queima}

As fenofases de E. gummiferum não foram alteradas com o regime de queimada (PC versus $\mathrm{PBM}$ ), sugerindo que essa espécie é pouco afetada por queimadas ocasionais. A maior frequência de fogo afetou, principalmente, a população de $C$. brasiliense, que sob regime mais intenso de queima não apresentou pico de brotação foliar e registrou retardamento da queda foliar. O fenômeno de retardamento da renovação das folhas em consequência de queimadas também foi observado em outra comunidade de Cerrado (FIGUEIREDO, 2008). De acordo com Williams et al. (1997), o controle na produção foliar ocorre por fatores endógenos, mas pode ser induzido por fatores externos (FRANCO et al., 2005), como ocorreu na PBM.

O regime de queima afetou negativamente as estruturas reprodutivas de $C$. brasiliense, refletindo em ausência de registro de floração e de frutificação 
na PBM. Além disso, a população de pequi não gerou estruturas reprodutivas na PBM no período reprodutivo subsequente e teve intensa produção na PC. A produção de frutos de Stryphnodendron adstringens também foi prejudicada no ano em que houve queimada e nos dois anos subsequentes (FELFILI et al., 1999). Estudando as fases reprodutivas de Qualea parviflora nas mesmas parcelas deste estudo, Palermo e Miranda (2012) observaram floração mais intensa na PBM do que na PC no ano de queimada, porém a mesma proporção de flores gerou frutos nas duas áreas. A diminuição ou ausência de estruturas reprodutivas diante da maior intensidade de queimadas pode ser consequência do investimento energético em novas estruturas vegetativas.

\section{CONCLUSÃO}

Caryocar brasiliense não apresentou queda foliar completa em nenhum momento durante este estudo, e sua estratégia reprodutiva (frutos carnosos em pequenas quantidades) é similar à de espécies sempreverdes. No entanto, Enterolobium gummiferum apresentou comportamento breve-decíduo e estratégia reprodutiva anual precoce. As variáveis duração das noites e precipitação média mensal apresentaram papel importante nas fenofases, especialmente na brotação, com folhas maduras e floração, e queda foliar e frutificação, respectivamente. O regime de queimada mais intenso não alterou as fenofases ou o potencial reprodutivo de E. gummiferum, mas retardou a troca das folhas e inibiu a produção de diásporos de Caryocar brasiliense. Tais alterações fisiológicas parecem ser um padrão e devem ser investigadas em estudos posteriores.

\section{AGRADECIMENTOS}

À Direção da RECOR e aos coordenadores do Projeto Fogo, em especial à professora Dra. Heloisa Sinatora Miranda; e ao Dr. Reuber Brandão, professor do Programa de Pós-Graduação em Ciências Florestais - UnB, pelos comentários e pelo auxílio nas análises dos dados.

\section{REFERÊNCIA}

ALENCAR, J. C. Fenologia de cinco espécies arbóreas tropicais de sapotaceae correlacionada a variáveis climáticas na reserva Ducke, Manaus, AM. Acta Amazonica, v.24, n.3/4, p.161-182, 1994.

Revista Árvore, Viçosa-MG, v.38, n.4, p.579-590, 2014
BADECK, F. W. et al. Responses of spring phenology to climate change. New Phytologist, v.162, n.2, p.295-309, 2004.

BATALHA, M. A.; MANTOVANI, W. Reproductive phenological patterns of Cerrado plant species at the Pé-de-Gigante Reserve (Santa Rita do Passa Quatro, SP, Brazil): a comparison between the herbaceous and woody floras. Revista Brasileira de Biologia, v.60, n.1, p.129-145, 2000.

BULHÃO, C. F.; FIGUEIREDO, P. S. Fenologia de leguminosas arbóreas em uma área de cerrado marginal no nordeste do Maranhão. Revista Brasileira de Botânica, v.25, n.3, p.361-369, 2002.

D’EÇA-NEVES, F. F.; MORELLATO, L. P. C. Métodos de amostragem e avaliação utilizados em estudos fenológicos de florestas tropicais. Acta Botanica Brasilica, v.18, n.1, p.99108, 2004.

FELFILI, J. M. et al. Estudo fenológico de Stryphnodendron adstringens (Mart .) Coville no cerrado sensu stricto da Fazenda Água Limpa no Distrito Federal, Brasil. Revista Brasileira de Botânica, v.22, n.1, p.1-10, 1999.

FERRAZ, D. K. et al. Fenologia de árvores em fragmento de mata em São Paulo, SP. Revista Brasileira de Biologia, v.59, n.2, p.305317, 1999

FIGUEIREDO, P. S. Fenologia e estratégias reprodutivas das espécies arbóreas em uma área marginal de cerrado, na transição para o semiárido no nordeste do Maranhão, Brasil.

Revista Trópica - Ciencias Agrárias e Biológicas, v.2, n.2, p.8-22, 2008.

FRANCO, A. C. et al. Leaf functional traits of Neotropical savanna trees in relation to seasonal water deficit. Trees, v.19, p.326-335, 2005.

GUILHERME, F. A. G. et al. Fenologia de Cybistax antisyphilitica (MART.) (Bignoniaceae) na região urbana de Jataí, Goiás. BioScience Journal, v.27, n.1, p.138-147, 2011.

Revista Árvore, Viçosa-MG, v.38, n.4, p.579-590, 2014 
KLINK, C. A. et al. O bioma Cerrado. In: SEELIGER, U.; CARDOSO, C.; BARBOSA., F. (Ed.). Os Sites e o Programa Brasileiro de Pesquisas Ecológicas de Longa duração2. Belo Horizonte: Universidade do Rio Grande, 2002. p.51-68.

KOVACH, W. L. Oriana - circular statistics for Windows, ver. 2.0 PentraethKovach Computing Services, 2006.

LENZA, E.; KLINK, C. A. Comportamento fenológico de espécies lenhosas em um cerrado sentido restrito de Brasília, DF. Revista Brasileira de Botânica, v.29, n.4, p.627-638, 2006.

LOPES, S. F.; VALE, V. S.; SCHIAVINI, I. Efeito de queimadas sobre a estrutura e composição da comunidade vegetal lenhosa do cerrado sentido restrito em Caldas Novas, GO. Revista Árvore, v. 33, n. 4, p. 695-704, 2009.

MIRANDA, H. S. et al. Queimadas de Cerrado: caracterização e impactos. In: CERRADOS, E. (Ed.). Cerrado: ecologia e caracterização. Planaltina: [s.n.].

MIRANDA, H. S. et al. Projeto fogo. In: RIBEIRO, M. L. (Ed.). Reserva Ecológica do IBGE. Biodiversidade terrestre. Rio de Janeiro: IBGE, 2011. v.1. p.162-177.

NEWSTRON, L. E. et al. Diversity of long-term flowering patterns. In: BAWA, K. S. et al. (Ed.). La Selva: ecology and natural history of a lowland tropical rain forest. Chicago: University of Chicago Press, 1993. p.142-160.

OLIVEIRA, V. Á. Solos da bacia do córrego Taquara. In: RIBEIRO, M. L. (Ed.). Reserva Ecológica do IBGE. Biodiversidade terrestre. Rio de Janeiro: IBGE, 2011. v.1. p.47-59.

PALERMO, A. C.; MIRANDA, H. S. Efeito do fogo na produção de Qualea parviflora MART. (Vochysiaceae) em cerrado sensu stricto.

Revista Árvore, v.36, n.4, p.685-693, 2012.

PEREIRA, B. A. S.; FURTADO, P. P. Vegetação da bacia do córrego Taquara: coberturas naturais e antrópicas. In: RIBEIRO, M. L. (Ed.). Reserva Ecológica do IBGE. Biodiversidade Terrestre. Rio de Janeiro: IBGE, 2011. v.1. p.89-117.
PIRANI, F. R.; SANCHEZ, M.; PEDRONI, F. Fenologia de uma comunidade arbórea em cerrado restrito, Barra do Garças, MT, Brasil. Acta Botanica Brasilica, v.23, n.4, p.1096-1109, 2009.

RANGEL, T. F.; DINIZ-FILHO, J. A. F.; BINI, L. M. SAM: a comprehensive application for Spatial Analysis in Macroecology. Ecography, v.33, n.1, p.46-50, 2010.

RECOR. Banco de dados meteorologicos. Disponível em: < http://www.recor.org.br/ index.php/banco-dados/dados-meteorologicos)>. Acesso em: 10 out. 2012.

RECOR. A Recor no contexto das áreas protegidas. Disponível em: <http:// www.recor.org.br/>. Acesso em: 25 mar. 2013.

ROCHA, H. M. C.; SILVA, J. C. S. Germinação e viabilidade de Enterolobium gummiferum (Mart.) Macb. (Leguminosae) e Plathymenia reticulata Benth. (Leguminosae) In:REUNIÓM LATINO AMERICANA DE FISIOLOGIA VEGETAL, 2002, Punta Del-Este. Anais...Punta Del-Este: Sociedad Latino Amaricana de Fisiologia Vegetal, 2002

RODRIGUES, F. H. G. Influência do fogo e da seca na disponibilidade de alimentos para herbívoros do cerrado. In: MIRANDA, H. S.; SAITO, C. H.; DIAS, B. F. DE S. (Ed.). Impactos de queimadas em áreas de cerrado e restinga. Brasília: Universidade de Brasília, 1996. p.76-83.

SARTORELLI, P. A. R. et al. Rebrota após fogo de espécies arbóreas de diferentes grupos fenológicos foliares em cerrado. Revista Científica Eletronica de Engenharia Florestal, v. 6, n.10, 2007.

SATO, M. N.; MIRANDA, H. S. Mortalidade de plantas lenhosas do cerrado sensu stricto submetidas a diferentes regimes de queima. In: MIRANDA, H. S.; SAITO, C. H.; DIAS, B. F. S. (Ed.). Impactos de queimadas em áreas de cerrado e restinga. Brasília: Universidade de Brasília, 1996. p.102-111.

SILVA-JÚNIOR, M. C. 100 árvores do cerrado: guia de campo. Brasília: Rede de Sementes do Cerrado, 2005.

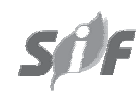

Revista Árvore, Viçosa-MG, v.38, n.4, p.579-590, 2014 
SILVÉRIO, D. V.; LENZA, E. Fenologia de espécies lenhosas em um cerrado típico no Parque Municipal do Bacaba, Nova Xavantina, Mato Grosso, Brasil. Biota Neotropica, v.10, n.3, p.205-216, 2010.

STAGGEMEIER, V. G.; DINIZ-FILHO, J. A. F.; MORELLATO, L. P. C. The shared influence of phylogeny and ecology on the reproductive patterns of Myrteae (Myrtaceae). Journal of Ecology, v.98, n.6, p.1409-1421, 2010.
VILELA, G. F.; CARVALHO, D.; VIEIRA, F. A. Fenologia de Caryocar brasiliense Camb. (Caryocaraceae) no Alto Rio Grande, Sul de Minas Gerais. Cerne, v.14, n.4, p.317-329, 2008.

WILLIAMS, R. J. et al. Leaf phenology of woody species in a North Australian Tropical Savanna. Ecology, v.78, n.8, p.2542-2558, 1997.

ZAR, J. H. Biostatistical Analysis. 4.ed. Upper Saddle River: Prentice Hall, 1999. 663p. 